\title{
Le Serment d'Hippocrate et les femmes grecques
}

\section{Lydie Bodiou}

\section{(2) OpenEdition}

\section{Journals}

\section{Édition électronique}

URL : https://journals.openedition.org/clio/1467

DOI : 10.4000/clio.1467

ISSN : 1777-5299

Éditeur

Belin

\section{Édition imprimée}

Date de publication : 1 avril 2005

Pagination : 231-238

ISBN : 2-85816-781-8

ISSN : 1252-7017

\section{Référence électronique}

Lydie Bodiou, «Le Serment d'Hippocrate et les femmes grecques », Clio. Histoire, femmes et sociétés [En ligne], 21 | 2005, mis en ligne le 01 juin 2007, consulté le 23 avril 2022. URL : http:// journals.openedition.org/clio/1467 ; DOI : https://doi.org/10.4000/clio.1467

Ce document a été généré automatiquement le 23 avril 2022

Tous droits réservés 


\title{
Le Serment d'Hippocrate et les femmes grecques
}

\author{
Lydie Bodiou
}

\section{NOTE DE L'ÉDITEUR}

Les remerciements de l'auteur vont à Pierre Brulé, Frédéric Chauvaud et Claudine Leduc pour leur lecture attentive et leurs conseils.

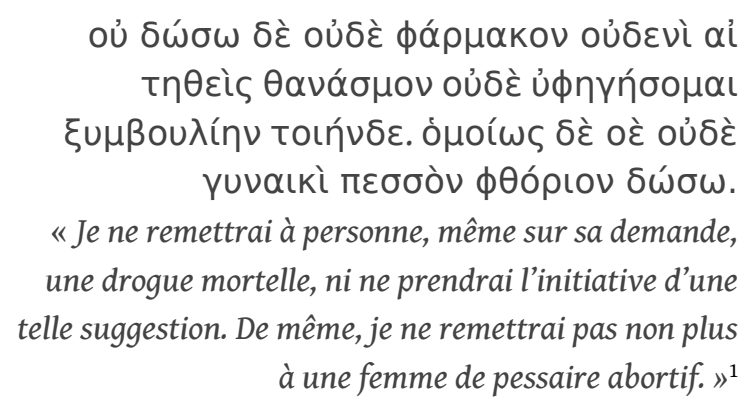

1 Le Serment d'Hippocrate, le texte le plus célèbre des écrits médicaux de l'Antiquité grecque, prend position en une seule phrase sur l'avortement. Les commentaires de ce passage ont été et sont toujours nombreux et donnent lieu à de multiples controverses. Les traductions proposées et les présupposés idéologiques ont orienté les différents commentateurs sur des pistes d'interprétation diamétralement opposées. Deux tendances se dégagent: pour les uns, il s'agit d'une condamnation pure et simple de l'avortement; pour les autres, le Serment interdit seulement un type de pratique abortive en raison de sa dangerosité. Mais peut-être convient-il aussi de s'interroger sur la perception que ces médecins avaient de l'avortement et de la contraception.

2 Avant de revenir à la matière du texte, il paraît nécessaire, afin de mieux en rendre compte, de fixer le contexte social, médical et gynécologique dans lequel cet engagement du Serment pouvait être pris par les médecins. Il importe de mieux comprendre d'abord ce qu'était l'obligation de maternité pour les femmes grecques, 
ensuite de s'attacher aux conceptions de l'époque en matière de contraception et d'avortement - et de bien les distinguer -, enfin de saisir la position du médecin, auquel les maris demandent que l'épouse soit toujours potentiellement féconde - c'est-à-dire par défaut, en vie - en même temps qu'ils refusent couramment d'élever des enfants. En effet, dans cette société où les femmes ne peuvent que difficilement se dérober au devoir de reproduction, l'enfant est souvent celui de trop, surtout si c'est une fille. La contraception et l'avortement sont d'un usage courant, des preuves figurant dans les traités gynécologiques du corpus hippocratique ${ }^{2}$ :

Quand la femme est affectée d'une grande plaie à la suite de l'avortement (trôsmos) ou quand la matrice a été ulcérée par des pessaires âcres, ce qui arrive, vu tant de pratiques et de traitements que les femmes font de leur chef; ou quand le fotus étant chassé par l'avortement (...), la malade, si elle est traitée promptement guérira, mais restera stérile ${ }^{3}$.

3 Ces pratiques contraceptives et abortives dissimulées relèvent de la communauté des femmes « qui se disent (les choses) entre elles » ${ }^{4}$. Nul doute que consulter un médecin constituait l'ultime recours, alors que tout ou presque avait été tenté auprès des sagesfemmes, les fameuses maiai, avorteuses, nourrices, femmes aux savoirs empiriques, aux remèdes de « bonnes femmes $»^{5}$.

Les traités gynécologiques hippocratiques ne se contentent ni de décrire les pathologies féminines liées à de telles pratiques, ni les thérapies adéquates, ils se terminent le plus souvent par des catalogues de recettes placés en annexes aux allures d'interminables listes de remèdes ${ }^{6}$. Des diurétiques, des emménagogues, des purgatifs utérins qui sont initialement prescrits pour "purger " la femme, "amener le sang des règles » et la rendre au plus vite féconde. S'ils ne sont pas spécifiquement prescrits comme abortifs ou contraceptifs, ils le deviennent. En effet, les médecins interviennent toujours après l'acte sexuel car pour eux, la conception n'est effective que lorsque le fœtus est animé. De la sorte, les premiers jours après l'acte, lorsqu'il n'existe que des semences inertes, le médecin les qualifie de "pertes ». En effet, «Les femmes confirment que la plupart des avortements ont lieu dans la première quarantaine $»^{7}$. Toutefois, « dans la plupart des cas, les jours les plus significatifs sont le premier et le septième, tant pour les maladies que pour le fœutus; en effet, la plupart des avortements (trôsmoi) se produisent ces jours-là ». Mais en réalité, à ce moment-là «Cela se nomme non pas avortements (trôsmoi) mais pertes (ekrusies) ॥ ${ }^{8}$. Les prescriptions médicales visent donc à expulser au plus tôt le sperme car «si une femme doit devenir enceinte, le sperme ne sort pas mais reste dans la matrice $»^{9}$. Aussi le médecin hippocratique peut-il prescrire et décrire une technique contraceptive :

Une femme que je connais avait une chanteuse renommée ayant commerce avec les hommes; il ne fallait pas qu'elle devînt enceinte, pour ne pas perdre de sa valeur. Cette chanteuse avait entendu ce que les femmes se disent entre elles: que si une femme doit devenir enceinte, le sperme ne sort pas mais reste dans la matrice. (..). Dès qu'elle s'aperçut que le sperme ne sortait pas; elle le dit à sa maitresse et le propos vint jusqu'à moi. Et moi, j'invitai à sauter en faisant aller ses talons jusqu'à ses fesses. Elle l'avait déjà fait sept fois, lorsque le sperme coula à terre en faisant un bruit $^{10}$.

5 Il y a là assurément une prescription médicale, une méthode clairement énoncée et une issue certaine qui, pour ce médecin, ne semble pas laisser la place à l'échec. Mais il précise au préalable qu'il s'agit « d'un sperme de six jours ». Puisque la conception n'a lieu que plus tard, lorsque le sperme masculin a agi sur la semence féminine en la coagulant ${ }^{11}$, il s'agit bien ici pour ce médecin d'une pratique contraceptive, empêchant 
le mélange des semences féminines et masculines à l'intérieur de l'utérus. On peut se demander d'ailleurs si les médecins, comme les femmes, n'étaient pas enclins à privilégier les «pertes ». Par souci déontologique pour les hommes de l'Art, conscients de la dangerosité des techniques abortives. Par intérêt et «bon sens » pour les femmes qui souhaitent s'assurer de l'assistance du médecin, le laissant parfois dans le doute: "Avec le solstice d'été, la femme d'Achelos avorta le $6^{\mathrm{e}}$ jour; elle avait des vomissements, des frissons, des sueurs. Je ne sais de combien de mois était l'enfant. Elle dit aussi avoir avorté au bout de 20 jours d'un autre enfant qui était mâle. Disait-elle vrai ? Je l'ignore $»^{12}$.

6 Au bout de quelques semaines, « la semence, venue de toutes les parties de l'homme et de la femme pour la formation de l'être humain et tombée dans la matrice de la femme, s'est coagulée : avec le temps en est issu un être humain développé» ${ }^{13}$, il ne peut plus être alors question d'expulser une semence, mais bel et bien de détruire un embryon animé. Il s'agirait pour ces médecins, d'un avortement. Dès lors si les médecins prescrivent nombre de recettes "expulsives » qui visent à chasser un embryon, c'est parce qu'ils supposent qu'il est mort, anormal ou putréfié et le précisent avant de détailler le mode opératoire et les ingrédients nécessaires. Par exemple: "Pour détruire et chasser l'embryon (embruon) qui ne faut aucun mouvement $\aleph^{14}$, ou encore «Infusion pour la matrice, propre à chasser l'embryon, s'il est mort : piler du safran, verser de la grasse d'oie, passer, infuser dans la matrice, et l'y laisser aussi longtemps que possible $»^{15}$. Mais rien n'indique, ni ne contredit d'ailleurs, que les médecins interviennent pour interrompre une grossesse à partir du moment où la femme a senti l'enfant bouger en elle. Toutefois, ils sont conscients de la dangerosité de leurs médications :

Les avortements (phthorai) sont plus pénibles que les accouchements. En effet, il n'est pas possible qu'il n'y ait pas de violence dans l'expulsion (phtharênai) de l'embryon, soit par un purgatif, soit par une boisson, soit par un aliment, soit par des pessaires, soit par tout autre cause ${ }^{16}$.

7 C'est en fonction de ces risques, et assurément de cela seulement, que le Serment donne une limite restrictive à ses prescriptions.

8 Les gynécologies du corpus incitaient les femmes à la procréation, en insistant sur les effets positifs que les grossesses répétées apportaient à leur santé, relayant ainsi les attentes des maris et/ou de la cité. La plupart des prescriptions proposées visent à vider le corps de la femme et à "faire revenir les règles ", signe patent que la femme est en capacité de concevoir à nouveau. L'observation des ingrédients utilisés ${ }^{17}$ atteste une présence importante de produits toxiques et vénéneux qui agissent violemment sur l'organisme de la femme, entraînant des irritations, des inflammations, des hémorragies. Certes le sang coule, le fotus est expulsé, mais les dégâts occasionnés sont parfois irréversibles. Le médecin le concède : "Quand même elle en réchapperait, elle sera stérile ${ }^{18}$. Ce fléau qu'est la stérilité prive les femmes de leur capacité à engendrer, forme de reconnaissance que la société grecque leur accorde. Aussi les médecins affichent-ils leur préoccupation et tentent-ils d'y remédier. Pour preuve, ils ont intitulé l'un de leurs traités Des femmes stériles ${ }^{19}$. Encore faudrait-il savoir si nombre d'entre elles étaient initialement infertiles ou si elles le sont devenues après avoir tenté seules de se « débarrasser» de cet enfant de trop ou après avoir utilisé la pharmacopée des médecins hippocratiques. Sans doute un peu des deux et successivement : l'ampleur du fléau était ainsi décuplée. 
C'est dans ce contexte de menace qui pèse sur la vie des femmes qu'il faut replacer la lecture du Serment : "Je ne remettrai à personne, même sur sa demande, une drogue mortelle, ni ne prendrai l'initiative d'une telle suggestion. De même, je ne remettrai pas non plus à une femme de pessaire abortif ». La difficulté de l'interprétation tient à la structure interne de la phrase et au parallélisme des deux propositions suggéré par le terme homoios. Ce terme signifie en effet « de la même manière ", " de façon similaire ". La première proposition justifie la prohibition de toute intervention du médecin, l'interdiction de donner la mort à un malade, même à sa demande. La seconde proposition, introduite par cet adverbe fort («de même»), interdit au médecin de prescrire délibérément un procédé abortif, sans doute pour les mêmes raisons, car pouvant entraîner la mort de la patiente. Toutefois, en raison de l'utilisation du terme homoios, il a pu sembler curieux à certains ${ }^{20}$, que le médecin introduise une restriction dans la seconde partie de sa phrase ${ }^{21}$. Ainsi a-t-on pu conclure que le Serment visait à proscrire tous les types d'avortement, comme il interdisait tous les médicaments jugés mortels. Mais le grec est précis et sans équivoque, pesson phthorion signifie pessaire abortif. Il s'agit de suppositoires vaginaux, de tampons de laine imbibés de substances irritantes diluées le plus souvent dans une base huileuse ou graisseuse et placés dans la matrice. Une des raisons de l'interdiction de ce type de pessaires trouve sans doute son origine dans sa dangerosité, provoquant l'évacuation souhaitée de l'embryon, mais aussi la mort de la femme. Ainsi cette interdiction des pessaires répond-elle au même souci déontologique que la première proposition de la phrase : préserver la santé et la fécondité de la femme ${ }^{22}$. Il ne s'agirait donc pas d'une interdiction des pratiques abortives, puisqu'elles ne sont pas pensées comme telles, mais seulement d'une recommandation éthique, le médecin ne souhaitant pas être complice du suicide de la patiente ; au plus sera-t-il le témoin de sa mort si elle se l'est administrée seule.

Les femmes grecques se mutilent et meurent d'avoir des enfants, mais aussi de ne pas pouvoir en avoir et sûrement de ne pas en vouloir. Le Serment en interdisant la prescription des pessaires abortifs, sans doute les plus dangereux, tente de limiter les risques qui menacent la santé et la vie des femmes. Parfois témoins, parfois complices et souvent impuissants, les médecins hippocratiques sont aussi spectateurs des pratiques féminines «car elles disent tout ce qu'elles savent et elles continueront à le faire, car on ne saurait ni en fait ni en parole, les persuader qu'elles connaissent quelque chose de mieux que ce qui se passe dans leurs corps $»^{23}$.

\section{BIBLIOGRAPHIE}

BRULÉ Pierre, 2001, Les femmes grecques à l'époque classique, Hachette.

CARRICK Patrick, 1985, Medical ethics in Antiquity : philosophical perspective on abortion and euthanasia, Dordrecht, Reidel.

DEAN-JONES Lesley-Ann, 1996, Women's bodies in classical greek science, Oxford, Clarendon Press. 
DEMAND Nancy, 1994, Birth, death and motherhood in classical Greece, Baltimore, The Johns Hopkins University Press.

DUCATILLON Jeanne, 2001, « Le Serment d'Hippocrate, problèmes et interprétations », Bulletin de l'Association G. Budé, p. 34-61.

FONTANILLE Marie-Thérèse, 1997, Avortement et contraception dans la médecine gréco-romaine, Laboratoires Searle, Paris.

JOUANNA Jacques, 1992, Hippocrate, Fayard.

JOUANNA Jacques, MAGDELAINE Caroline, 1999, Hippocrate. L'art de la médecine, GF Flammarion.

KAPPARIS Konstantinos, 2002, Abortion in the ancient world, London, Duckworth.

KING Helen, 1998, Hippocrates' woman. Reading the female body in ancient Greece, London \& NY, Routledge.

LAALE H. Willer, 1993, « Abortion in greek antiquity : Solon to Aristotle», I et II, Classical and modern literature, 13. 2, p. 157-166 et 13.3, p. 191-210.

MURRAY James Stuart, 1991, " The alleged prohibition of abortion in the Hippocratic oath ", Echos du Monde Classique, XXXV, 10, p. 293-311.

NARDI Enzo, 1971, Procurato aborto nel Greco-romano, Milan, A. Giuffrè Editore.

RIDDLE John M., 1991, « Oral contraceptives and early term abortifacients during classical Antiquity and the Middle Ages», Past and Present, 132, p. 3-32.

-, 1992, Contraception and abortion from the ancient world to the Renaissance, Cambridge, Harvard University Press.

-, 1997, Eve's herbs : a history of contraception and abortion in the West, Cambridge, Harvard University Press.

TEMKIN Owsei \& C. LILIAN (eds.), 1967, Ancient medecine. Selected papers of Ludwig Edelstein, Baltimore, The John Hopkins Press.

\section{NOTES}

1.* Texte de J.-L. Heiberg, 1927, Hippocratis Opera, Corpus Medicorum Graecorum, I, 1, Berlin-Leipzig, Teubner, in Ducatillon 2001. Traduction in Jouanna et Magdelaine 1999 : 69-71.

2. Les traités hippocratiques gynécologiques et embryologiques : Maladies de femmes I et II, Femmes stériles, Nature de la femme, Superfétation, traductions E. Littré, 1839-1861 et Maladies IV, Génération, Nature de l'enfant, Du fœtus de 8 mois, traductions R. Joly, 1970, Belles Lettres. Pour une étude complète des traités, Jouanna 1992.

3. Maladies de femmes, I, 67 (L. VIII, 140-142).

4. Nature de l'enfant, 13, 1 (L. VII, 490).

5. Dean-Jones $1996: 26-31$.

6. Nous sommes redevables pour cet article à Fontanille 1977, qui offre de nombreuses pistes de recherches.

7. Du fœtus de 8 mois, 4,2 (L. VII, 442, 12-444, 4).

8. Du fœtus de 8 mois, 9, 2 (L. VII, 448, 1-8). Ceci est confirmé par Aristote, Histoire des animaux, VII, 3, 583b et Génération des animaux, III, 9, 758b, trad. P. Louis, Belles Lettres. 
9. Nature de l'enfant, 13, 1 (L. VII, 490).

10. Nature de l'enfant, 13, 1-2 (L. VII, 490-492).

11. Femmes stériles, 222 (L. VIII, 128).

12. Epidémies, 4, 6 (L. V, 146, 9-12).

13. Maladies IV, 32 (L. VII, 542).

14. Maladies des femmes, 1,78 (L. VIII, 188) : « Pour détruire et chasser le fœtus qui ne fait aucun mouvement : alun fendu d'une drachme, myrrhe autant, hellébore noir, trois oboles, bien broyer dans du vin noir, faire des glands et appliquer jusqu'à ce qu'ils se dissolvent peu à peu ».

15. Maladies des femmes, I, 78 (L. VIII, 188).

16. Maladies de femmes, I, 72 (L. VIII, 150-152).

17. Cf le catalogue des recettes et l'analyse précieuse in Fontanille 1977 : 75-149.

18. Maladies des femmes, I, 65 et 3. Mais voir aussi Maladies des femmes, 1, 67, 63, 85 ; II, 131, 134. Nature de la femme, 6-7, 14 et 21.

19. L. VIII, 213-249.

20. Ici les commentateurs suivent la proposition de L. Edelstein qui traduit pesson phthorion par « remède abortif ", terme générique qui condamne la pratique. Pour L. Edelstein, il s'agit d'une addition pythagoricienne tardive au corpus, qui reflétait davantage des préoccupations d'ordre philosophique que médical. Voir Temkin 1967.

21. Carrick $1985: 84-85$.

22. Kapparis 2002.

23. Du fœtus de 8 mois, 4 (L. VII, 440-442).

\section{RÉSUMÉS}

Le Serment d'Hippocrate, sans doute le texte que la postérité a retenu comme le plus fameux des écrits médicaux de l'Antiquité grecque, prend en une phrase position sur l'avortement: "Je ne remettrai pas non plus à une femme de pessaire abortif ". Les commentaires de ce passage du Serment ont été et sont toujours nombreux et donnent lieu à de multiples controverses. Mais une lecture littérale permet d'affirmer qu'il ne s'agit nullement d'une interdiction pure et simple de l'avortement, mais seulement d'une interdiction d'un type de pratique abortive en raison de sa dangerosité. Car les femmes grecques se mutilent et meurent d'avoir des enfants, mais aussi de ne pas pouvoir en avoir et sûrement de ne pas en vouloir. Les médecins le savent.

The Hippocratic oath, undoubtedly the most famous text that posterity has retained medical writings of Greek Antiquity, presents the following position on abortion: "Nor will I give to a woman an abortive pessary». The comments around this statement have always been numerous and given rise to multiple controversies. But a literal reading makes it possible to affirm that it by no means prohibits abortion, purely and simply, rather it prohibits certain dangerous abortive practices. Because Greek women mutilated themselves and died to have children, but also in order not to be able to have them - and doctors knew this. 
INDEX

Index géographique : Grèce

Index chronologique : antiquité

Mots-clés : avortement, contraception, Hippocrate

\section{AUTEUR}

\section{LYDIE BODIOU}

Lydie BODIOU est maître de conférences d'Histoire grecque à l'Université de Poitiers, membre du laboratoire CRESCAM de Rennes. Sa thèse sur Le sang des femmes grecques (à paraître) dit assez où s'enracine son intérêt pour l'histoire des femmes et du corps. Elle a publié : « Chemins de femmes, chemins de sang en Grèce à l'époque classique », Lunes, $n^{\circ} 20$, juillet 2002 ; Au jardin des Hespérides. Histoire, société et épigraphie des mondes anciens, PUR, 2004. 